\title{
DEVELOPMENTAL BIOLOGY OF MARINE ORGANISMS
}

A Symposium organized by the Marine Biological Association and the Society for Experimental Biology, and held at the University of Plymouth, 19-21 April 1993

Abstracts of papers and posters presented

EDITED BY C. BROWNLEE

Marine Biological Association

\section{PAPERS}

\section{Calcium stores involvement during serotonin-induced meiosis reinitiation in ruditapes oocytes}

\author{
P. Guerrier, C. Leclerc-David and M. Moreau
}

UMR 49, Ecole Normale Supérieure de Lyon, 46 Allée d'Italie, 69364 Lyon Cédex 07, France. UMR 9925, Université Paul Sabatier, 118 Route de Narbonne, 31062 Toulouse Cédex, France. Stations Biologiques de Roscoff et de Concarneau, France

In contrast to the situation found in the bivalves Barnea candida and Spisula solidissima prophasearrested oocytes of Ruditapes philippinarum cannot be fertilized when extracted from the ovary. They must first undergo germinal vesicle breakdown (GVBD) under the influence of the neurohormone serotonin (5-HT) which drives them to a second block occurring in metaphase of the first maturation division. We used Fluo-3 to investigate the possibility that calcium was involved as a second messenger in controlling this first step in meiosis reinitiation. Our data showed that, in addition to 5-HT, ionophore, thapsigargin and the weak bases ammonia and procaine could induce prophase-arrested oocytes of Ruditapes to resume meiosis. Like 5-HT, thapsigargin and ammonia were found able to trigger an intracellular $\mathrm{Ca}^{2+}$ surge and GVBD even in the absence of external $\mathrm{Ca}^{2+}$. Loading the oocytes with BAPTA/AM or treating them with D-600 blocked maturation. In contrast, excess $\mathrm{KCl}$, which was effective on Barnea and Spisula, did not produce any significant intracellular $\mathrm{Ca}^{2+}$ transient or meiosis reinitiation, when added to Ruditapes prophase-arrested oocytes. These data suggest that intracellular $\mathrm{Ca}^{2+}$ stores are actually mobilized following binding of 5-HT to its receptors and that the absence of voltageoperated $\mathrm{Ca}^{2+}$ channels may account for the unfertilizability. of Ruditapes prophase-arrested oocytes.

\section{Starfish oocytes and sea urchin eggs, two models to study cell cycle control}

\author{
L. Meijer, A.C. Anderson, L. Azzi, B. Baratte, L. Diaz and G. Mordet \\ CNRS, Station Biologique, 29682 Roscoff Cédex, France
}

Starfish oocytes are naturally arrested in the late G2 of the first meiotic prophase. The follicle cell-derived hormone 1-methyladenine triggers the G2/M transition and the completion of 
proteins. It induces a decrease of cyclic AMP concentration and presumably the appearance of a yet unidentified intracellular second messenger. This leads to modifications of the phosphorylation

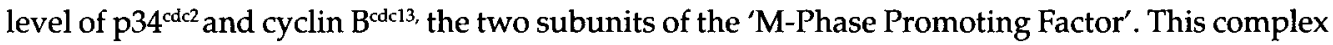
then displays kinase activity towards a variety of protein substrates.

Sea urchin eggs are naturally arrested in the G1 phase of the cell cycle. Fertilization triggers a series of highly synchronous mitotic divisions. Assembly and activation of the M-Phase Promoting Factor can also be observed as these cells proceed through the cell cycle. We illustrate the impact of these two models in our understanding of intracellular cell cycle control and discuss the upstream regulatory pathways controlling M-Phase Promoting Factor activation.

\title{
Fertilization calcium waves
}

Karl Swann and Michael Whitaker

MRC EET Unit, St George's Hospital Medical School, Cranmer Terrace, London, SW17 ORE and Department of Physiology, University College London, Gower Street, London, WC1E 6BT

Everyone is agreed that fertilization in deuterostomes is accompanied by a large intracellular calcium wave that triggers the onset of development, but we still do not know exactly how the calcium wave is generated. The question has two parts: how does interaction of sperm and egg initiate the calcium wave and how does the calcium wave spread across the cell. Two provisional answers are available to the first part of the question, one involving receptor-G protein interactions of the sort that mediate transmembrane signal transduction in somatic cells, the other injection of an activating messenger when sperm and egg fuse. Both these ideas are being actively pursued; the dialectic is productive, albeit no synthesis is in sight. We discuss their strengths and weaknesses. The second part of the question can now be much more precisely formulated thanks to the recent flush of interest in calcium waves in somatic cells: new ideas and new experimental tools are available. The work on somatic cells repays a debt to eggs, where the basic properties of calcium waves were first set out, ten years before they turned up in somatic cells.

\section{Release from the metaphase block in invertebrate oocytes: possible involvement of $\mathrm{Ca}^{2+} /$ calmodulin kinase III}

\author{
H. Abdelmajid, C. Leclerc-David, M. Moreau, P. Guerrier and A. Ryazanov
}

UMR 49, Ecole Normale Supérieure de Lyon, 46 Allée d'Italie, 69364 Lyon Cédex 07, France. UMR 9925, Université Paul Sabatier, 118 Route de Narbonne, 31062 Toulouse Cédex, France. State University of New Jersey, Rutgers Campus, Smith Hall, Newark, New Jersey 07102, USA

Oocytes of the prosobranch gastropod mollusc Patella, the bivalves Mytilus, or Ruditapes provide an excellent model to study metaphase-anaphase transition. They are naturally arrested in metaphase of the first maturation division, and their fertilization or artificial activation results in cyclin destruction and completion of meiosis. Using the fluorescent $\mathrm{Ca}^{2+}$ indicator dye Fluo-3, we showed that both fertilization or $\mathrm{KCl}$-dependent activation trigger a measurable transient increase in cytosolic free $\mathrm{Ca}^{2+}$ concentration. On the basis of our experiments we propose that cyclin $\mathrm{B}$ degradation and release from the metaphase block depend upon activation of $\mathrm{a} \mathrm{Ca}^{2+} /$ calmodulin dependent kinase susceptible to phosphorylate elongation factor 2 (eEF-2). Phosphorylation of this factor would lead to a transient arrest of protein synthesis resulting in disappearance of the short lived proteins which inhibit the cyclin B degradation pathway. In support of this hypothesis, we found the following: (1) eEF-2 kinase is present in oocytes of Patella, Mytilus and Ruditapes; (2) inhibition of protein synthesis by emetine releases these oocytes from their metaphase block; (3) calmodulin antagonist such as TFP and W7 can reversibly block 
from their metaphase block; (3) calmodulin antagonist such as TFP and W7 can reversibly block fertilization, $\mathrm{KCl}$, or $\mathrm{A} 23187$ dependent activations, but not the activation triggered by emetine; (4) fertilization produces a transient inhibition of protein synthesis.

\title{
Cytoplasmic calcium and fertilization in Fucus eggs
}

\author{
S.K. Roberts and C. Brownlee
}

Marine Biological Association, The Laboratory, Citadel Hill, Plymouth, PL1 2PB

Fertilization in a range of animal eggs is accompanied by a wave of elevated free cytoplasmic calcium propagating (probably by calcium-induced calcium release (CICR) from internal stores) through the egg. This wave is thought to play a central role in activation of the egg.

The marine brown alga, Fucus serratus, provides one of the few systems for the study of plant egg activation. A variety of calcium-sensitive fluorescent indicators reveal that cytoplasmic calcium elevation occurs during fertilization, but is small and variable in comparison to most animal systems and is not always detected, at least as an average global calcium elevation. Microinjection of appropriate concentrations of calcium (BAPTA) buffers can totally abolish any fertilization-induced changes in global calcium while not preventing fertilization-associated cell wall exocytosis or subsequent zygote development. Higher [BAPTA] does inhibit activation and greatly prolongs the duration of the fertilization potential. Tracer experiments suggest a role for calcium uptake across the plasma membrane during fertilization. Preliminary studies using a confocal microscope suggest no involvement of a wave of CICR. The data suggest that a large transient global elevation of cytoplasmic calcium is not necessary for Fucus egg activation, though local elevation resulting from influx across the plasma membrane is needed for early fertilization events.

\section{Surface glycoproteins and their role in egg-sperm recognition in Fucus}

\author{
J.A. Callow, C.R. Stafford, P.J. Wright and J.R. Green \\ School of Biological Sciences, University of Birmingham, Birmingham, B15 2TT
}

The distribution of glycoprotein antigens on the surface of Fucus eggs has been examined using several antibodies and lectins in conjunction with confocal laser scanning microscopy, immunogold electron microscopy and Western blotting. Different sets of surface glycoprotein antigens appear to be organized into distinct spatial domains, others are more uniformly distributed. The functional significance of these domains in fertilization has been explored using MAbs, Fab fragments and lectins. Results show that there is functional as well as spatial heterogeneity and in one of the glycoprotein sub-sets a $62 \mathrm{kDa}$ component has been identified as a putative receptor.

In parallel studies on sperm surface components, a KCl-solubilized fraction binds to egg plasma membranes and blocks fertilization species-specifically. Binding is blocked by sulphated polysaccharides. From the crude $\mathrm{KCl}$ extract a $60-70 \mathrm{kDa}$ fraction can be isolated by HPGF which binds to egg membranes and elicits cell-wall release from unfertilized eggs. The involvement of components of this fraction in egg activation are discussed.

\section{Polyphosphoinositide metabolism during sea urchin egg mitotic divisions}

\section{B. Ciapa* and D. Pesando ${ }^{\dagger}$}

'Laboratoire de Physiologie Cellulaire et Comparée, Faculté des Sciences, Parc Valrose, BP 71,06108 Nice, Cédex 02, France. ${ }^{\dagger}$ Unité INSERM 303, BP3, 06230 Villefranche-sur-Mer, France

It has been suggested that changes in intracellular free calcium occur during the cell cycle to control nuclear and cellular division. We have investigated whether this event could be related to 
variations in polyphosphoinositide metabolism. Eggs were fertilized in the presence of [32P]orthophosphate and the incorporation of this precursor into phosphatidylinositol phosphate (PIP) and phosphatidylinositol bisphosphate (PIP2) was measured. While [32P]-orthophosphate accumulation into eggs accelerated with time, its incorporation in inositol-containing lipids fluctuated during the first four cell cycles. Peaks of incorporation of the precursor into PIP2 occurring during the first cell cycle corresponded to morphologic events (pronuclear migration, streak stage and cleavage) and to transient increases of intracellular free calcium reported by others. The subsequent peaks corresponded to the second, third and fourth cleavages, and still occurred in eggs incubated in the presence of inhibitors of protein synthesis, emetine and nordidemenin, which arrest mitotic divisions during the first and the second cell cycle respectively. We observed peaks of incorporation of 32P into PIP before the second, third and fourth cleavages. The incorporation of $32 \mathrm{P}$ into PIP dropped after one hour of treatment with emetine or nordidemenin, but with a suggestion of fluctuations during the second and the third cell cycles. We conclude that fertilization induces PPI metabolism to oscillate independently of protein synthesis, the intensity of the oscillations being, however, dependent on protein synthesis. Our observations reinforce a hypothesis raised previously by Twigg et al., pointing to the PPI metabolism as an important component of the cell cycle regulation in sea urchin eggs.

\section{Establishment and reorganization of cytoplasmic domains in ascidian eggs following fertilization}

C. Sardet* , J. Speksnijder ${ }^{\dagger}$, A. Biollot ${ }^{*}$, B. Flannery*, C. Rouviere*, F. Roegiers* \& J. Davoust ${ }^{\ddagger}$

*Unité de Biologie Cellulaire Marine, URA 671 CNRS/UPMC, Observatoire Océanologique, Station Zoologique, Villefranche-sur-Mer 06230, France. ${ }^{\dagger}$ Hubrecht Laboratory, Netherland Institute for Developmental Biology, Uppsalalaan 8, 3584 CT Utrecht, The Netherlands. ${ }^{\ddagger}$ Centre d'Immunologie de Marseille-Luminy, CNRS/INSERM, Marseille 13288, France

Ascidian eggs undergo dramatic cortical and cytoplasmic reorganizations between fertilization and first cleavage that participate in the establishment of the dorso-ventral and antero-posterior axes of the embryo.

We show that in the egg of the ascidian, Phallusia mammillata, the cortical contraction triggered by the fertilizing sperm starts first on the side of sperm entry. As a consequence, the cortical contraction is asymmetric with respect to the pre-existing animal-vegetal axis, and leads to the transient formation of a microfilament-rich constriction covered with microvilli that is close to, but generally not coincident with, the vegetal pole. This vegetal-contraction pole marks the site of gastrulation and thus the future dorsal side of the embryo.

The cortical contraction causes the basket-shaped mitochondria-rich subcortical cytoplasm (myoplasm) to concentrate in the vegetal-contraction pole area by the time the first polar body is extruded ( $4 \mathrm{~min}$ ). The myoplasm is relocated after the second polar body is formed $(24 \mathrm{~min})$ in two distinct subphases. We have now observed that the myoplasm first folds in its centre near the vegetal-contraction pole and moves slowly in an oscillatory fashion toward the spherical centrosomal region that forms around the male pronucleus. The myoplasm then tears as the male MTOC duplicates and the corresponding centrosomal area acquires the form of a disc. After tearing, the bulk of the myoplasm tightly adhering to the male pronucleus localizes with a smooth fast motion towards the future posterior pole of the embryo. A part of this myoplasm accompanies the adjoining male and female pronuclei towards the egg's centre where syngamy occurs. The remaining subcortical myoplasm forms a subequatorial girdle around the egg. Our observations with confocal and electron microscopy indicate that during these relocations the distinct cytoplasmic domains identified in the ascidian egg move without mixing their 
characteristic organelles and cytoskeletal constituents. We propose a description of the movements and forces at work in the process of ooplasmic segregation in ascidians.

\title{
Saving the children: defence mechanisms of marine embryos \\ David Epel \\ Hopkins Marine Station of Stanford University, Pacific Grove, CA 93950, USA
}

Much is known about how the multicellular adult organism defends itself against such stresses as xenobiotics and pathogens. But how can an embryo, with its few undifferentiated cells, achieve similar defences? I shall review two lines of research on such embryonic defence mechanisms.

We find that xenobiotic resistance (i.e. resistance to natural and man-introduced toxins) in the embryos of the mud-dwelling echiuroid worm, Urechis caupo, is carried out via the activity of an ATP-dependent multixenobiotic transporter present in the plasma membrane of oocytes and embryos. This transporter is immunologically similar to the multidrug resistance transporter found in mammalian cells. The Urechis transporter activity results in the efflux out of the cell of a variety of moderately hydrophobic compounds. Substrates for the xenobiotic transporter are also present in the sediments, indicating that the transporter can function to protect the embryo from toxic products present in its environment. The Urechis embryo can develop in water associated with the sediments, but embryos which do not have this transporter (such as sea urchin embryos) exhibit abnormal development.

Protection through symbiotic bacteria might be the role of bacteria associated with the egg case of the squid Loligo opalescens. These bacteria apparently come from the accessory nidamental gland, which houses symbiotic bacteria and is connected to the ovary. At least four species of bacteria are associated with the accessory nidamental gland. Research on the role of these bacteria is described.

\section{Fibronectin and related substances in sea urchin eggs and embryos}

\author{
Y. Yokota*, V. Matranga ${ }^{\dagger}$, F. Zito ${ }^{\dagger}$, M. Cervello ${ }^{\dagger}$ and E. Nakano ${ }^{\dagger}$
}

*Biological Laboratory, Aichi Prefectural University, Mizuho, Nagoya 467, Japan. ${ }^{+}$Istituto di Biologia dello Sviluppo, Consiglio Nazionale delle Ricerche, Via Archirafi 20, 90123 Palermo, Italy

The extracellular matrix (ECM) plays a key role in morphogenesis during embryonic development of various organisms. In the sea urchin two ECM proteins with molecular weights of 180,000 and 115,000 were identified and investigated. The ECM protein with a molecular weight of 180,000 appeared to correspond to fibronectin of vertebrates and the ECM protein with a molecular weight of 115,000 seemed to be a nectin specific to echinoderms. Fibronectin and the nectin with a molecular weight of 115,000 were separable from each other by affinity chromatography on gelatin-Sepharose. Polyclonal antibodies against fibronectin and the nectin specific to echinoderms were raised to analyse phylogenic relationships of the nectins and their biological roles. Immunoblotting analysis of fibronectin and the nectin, and immunoflourescent microscopical observation of developing embryos were carried out in the five species of Mediterranean and Japanese sea urchins, Paracentrotus lividus, Hemicentrotus pulcherrimus, Anthocidaris crassispina, Pseudocentrotus depressus, Temnopleurus hardwicki. Fibronectin and the nectin were regarded to be different molecular species from each other, since they showed none of the common epitopes. The immuno-cross-reactivity of the nectin with a molecular weight of 115,000 presumably has some relevance to its behaviour in affinity chromatography on gelatinSepharose. Although this nectin is localized evenly in the unfertilized egg, it is translocated to the 
surface area of embryos and secreted into the hyaline layer after fertilization. In blastulae it becomes localized in the area where the ingression of mesenchymal cells takes place. Experiments on the embryos cultured in the presence of Fab against the nectin specific to echinoderms, and localization of the antigen, suggested the hypothesis that this nectin may play an important role in migration of mesenchymal cells and formation of spicules.

\title{
Intracellular $\mathrm{pH}$ gradients in embryos of fucoid algae
}

\author{
D.L. Kropf and B.C. Gibbon \\ Department of Biology, University of Utah, Salt Lake City, UT 84112, USA
}

Intracellular $\mathrm{pH}\left(\mathrm{pH}_{\mathrm{i}}\right)$ is an important regulator of early development in animals, but little is known about its function in plant embryogenesis. We have initiated a study of pHi in the first two zygotic cell cycles in the marine brown alga, Pelvetia. Using $\mathrm{pH}$-sensitive microelectrodes, we have discovered that a $\mathrm{pH}$ gradient is present along the growing rhizoid cell. The tip cytoplasm is $\mathrm{pH} 7.2$ whereas the basal cytoplasm of the same cell is $\mathrm{pH} 7.5$. Between these positions the gradient is nearly linear. The physiological significance of the $\mathrm{pH}$ gradient was investigated by adding propionic acid, a membrane permanent weak acid. At millimolar concentrations propionic acid caused substantial cytoplasmic acidification, but when added to sea-water at $500 \mu \mathrm{M}$ it abolished the $\mathrm{pH}$ gradient without significantly altering $\mathrm{pH}_{\mathrm{i}}$. Rhizoid growth was also inhibited by $500 \mu \mathrm{M}$ propionic acid, demonstrating a correlation between the intracellular gradient and growth. To our knowledge, this is the first quantitative description of $\mathrm{pH}$ gradients in tip-growing cells.

\section{Plasma membrane channels, cytoplasmic calcium and polarity in the fucoid zygote}

\section{F. Berger, A.R. Taylor and C. Brownlee \\ Marine Biological Association, The Laboratory, Citadel Hill, Plymouth, PL1 2PB and ENS Lyon, 46 Allée d'Italie, 69364 Lyon Cédex 07, France}

The zygote of the marine brown alga Fucus polarizes according to environmental cues before the first cell division, which then determines a rhizoid and a thallus cell. Laser scanning ratio confocal microscopy has revealed localized $\mathrm{Ca}^{2+}$ elevation at the growing Fucus rhizoid apex. Locally elevated $\mathrm{Ca}^{2+}$ can be visualized using confocal microscopy well before rhizoid germination, suggesting a causative role for the $\mathrm{Ca}^{2+}$ elevation. We hypothesize that the localized $\mathrm{Ca}^{2+}$ elevation originates from localized channel activity.

Direct analysis of channel activity and distribution in developing zygotes has required development of a non-enzymatic method for localized access to the plasma membrane. We have used a finely focused UV laser to ablate selected regions of the cell wall. Careful adjustment of the osmoticum can enable extrusion of plasma membrane-bound cytoplasm for patch-clamping. High resistance seals can be formed routinely on localized cytoplasmic extrusions. To date, a range of channel types has been identified in the apical plasma membrane. Preliminary results indicate higher average channel activity at the growing apex.

\section{Characterization of proteins that may be involved in polarity establishment and polarized cell growth in Fucus embryos}

\author{
Brad Goodner and Ralph Quatrano \\ Biology Department, University of North Carolina, Chapel Hill, NC 27599-3280, USA
}

We are using the brown alga Fucus as a model to study polar axis establishment and polarized cell growth in plants. The first cell division of the Fucus zygote is asymmetrical and results in two 
cells which differ dramatically in their developmental fates. Previous work in our laboratory indicated a role for the actin cytoskeleton in setting up the polar axis and a role for the actin cytoskeleton and the cell wall in stabilizing that axis. More recently a cell wall protein has been identified that has properties similar to those of vitronectin, an extracellular matrix protein found in animals. This work is reviewed and an Axis Stabilizing Complex model presented. To identify other proteins involved, in polarity establishment and polarized cell growth we have taken an approach based on the hypothesis that many of the mechanisms involved in these processes, and the proteins involved have been conserved during eukaryotic evolution. Budding in the yeast Saccharomyces cerevisiae, an organism very distinct phylogenetically from plants, shares many similarities with the first cell division in Fucus. Genetic and molecular studies in other laboratories have identified many proteins involved in selecting the site of budding, setting up the polar axis, and bud growth. We have used some of the same antibody and gene probes to look for homologous proteins in Fucus. We have concentrated on two families of proteins. Members of the one family, the ras-like proteins, are involved at each stage of the budding process. The other family contains the so-called neck filament proteins. These proteins were first described in $S$. cerevisiae as subunits of a unique cytoskeletal system of $10-\mathrm{nm}$ filaments localized around the site of bud growth. Results from Western blot, PCR cloning, Southern blot and Northern blot analyses are discussed with respect to these two protein families in Fucus.

\title{
Embryonic axis specification and microtubule-based movements in the ctenophore Beroe ovata
}

\author{
E. Houliston, D. Carre, C. Rouviere and C. Sardet \\ Unité de Biologie Cellulaire Marine, URA 671 CNRS/UPMC, Observatoire Océanologique, Station \\ Zoologique, Villefranche-sur-Mer 06230, France
}

The Beroe ovata egg has no pre-established developmental axis, rather the future organization of the animal depends upon the position of the first mitosis, which in turn depends on the random position of sperm entry. After first cleavage the embryo exhibits a stereotypical cleavage pattern and classical mosaic development. We have been examining the cellular processes involved in reorganizing the egg cytoplasm and defining the embryonic axes, exploiting the exceptional clarity and large size of the egg. Fertilization in Beroe is polyspermic and is followed by migration of the female pronucleus to join one of the stationary male pronuclei. Pronuclear migration takes place just below the plasma membrane over long distances (up to $1 \mathrm{~mm}$ ) and can be followed easily by fluorescence or DIC video microscopy. The migration is microtubuledependent, with giant asters nucleated by sperm centrioles directing the movement. We have analysed the variations in the speed and trajectories of female pronuclei under different conditions using automated image processing. During the hour between pronuclear fusion and first mitosis a wave of cytoplasmic reorganization sweeps across the egg. It starts from the zygote nucleus and progresses along the future oral-aboral axis of the animal. Time-lapse video microscopy of eggs treated with cytoskeletal inhibitors reveals that this wave has microtubule and microfilamentbased components. Confocal microscopy of live eggs injected with rhodamine-tubulin shows that the giant sperm asters disappear successively, starting closest to the zygote nucleus. This appears to reflect the propagation of mitotic activity across the egg, with resultant regional modulation of microtubule dynamics. 


\title{
Maternal genes controlling ascidian development and evolution
}

\author{
W.R. Jeffery, K.W. Makabe and B.J. Swalla \\ University of California, Davis, Department of Zoology and Bodega Marine Laboratory, Bodega Bay, \\ CA 94923, USA and Station Biologique, Roscoff, France
}

Ascidian development is controlled in part by maternal factors stored in the unfertilized egg. We have studied closely-related ascidian species with different modes of development to identify these maternal factors. Two Molgula species found in sand flats at Roscoff, France, are closely related, but have different larval forms. Molgula oculata develops into a conventional tailed (or urodele) larva with a neural sensory cell, a notochord, and flanking bands of striated muscle cells. In contrast, Molgula oculata embryos develop into a tail-less (or anural) larva lacking these urodele larval features. Remarkably, some of the lost urodele features, including the neural sensory cell and a short tail with notochord, can be restored in hybrid embryos produced by fertilizing eggs of the anural species with sperm of the urodele species. The critical sperm component in this evolutionary restoration is the nuclear genome, suggesting that elimination of certain urodele larval features is caused by loss of function mutations in zygotic genes. Muscle cell differentiation, however, is not restored in these hybrid embryos, suggesting that this urodele feature is governed by maternal genes. To identify candidates for maternal factors that mediate tail muscle development, we have devised a subtractive procedure to clone genes expressed in oocytes of the urodele but not the anural species. Three different maternal urodele (mUro) clones have been isolated which encode regulatory factors expressed preferentially during oogenesis of the urodele species. The mUro 1 clone encodes a tyrosine kinase, mUro 2 a member of the bZIP family of DNA binding proteins, and mUro 11 a nuclear protein with $\beta$ strand 'saddle' and zinc finger DNA binding motifs. We propose that the mUro genes function in a pathway leading to tail muscle differentiation. In addition, the down regulation of the mUro genes in the anural species may function as a switch that converts a urodele to an anural species during evolution.

\section{Neuropeptides and development in echinoderm larvae}

\section{Claire Moss, Robert Burke* and Michael Thorndyke \\ Department of Biology, Royal Holloway, University of London, Egham, Surrey, TW20 OEX *Department of Biology, University of Victoria, Victoria, BC, V8W2Y2, Canada}

Echinoderms have for many years provided excellent model systems for the study of development, with most investigations focusing on very early developmental stages, constructing cell lineage histories and fate maps. Later stages are also of interest with regard to feeding and behavioural mechanisms, and in particular the phenomenon of metamorphosis in indirectly developing echinoderms. At this time much of the larval tissue degenerates and the adult develops from the so-called adult rudiment or vestibule, an invagination which comprises a patch of larval ectoderm together with cells derived from the coelomic pouches. Remarkably the larval nervous system completely disintegrates to be replaced by a new system arising from larval ectoderm.

Until now maps of the distribution of transmitters within the larval nervous system have been mainly limited to those describing the expression of serotonin in echinoid and asteroid larvae. In adults, serotonin has proved difficult to detect and, since one of the most interesting features of echinoderm development is the emergence of the adult nervous system at metamorphosis, it would be useful to identify a transmitter expressed by both larval and adult systems.

Recently we have isolated a new family of regulatory neuropeptides from echinoderms, the 
SALMFamides, which have an extensive distribution in the nervous system of all adult species studied so far. We have used specific antibodies raised against synthetic fragments of these native peptides to map peptidergic networks in echinoid and asteroid larvae with a view to following development through metamorphosis.

In larvae of the echinoid, Dendraster excentricus, and the asteroids, Asterias rubens and Pisaster ochraceus, SALMFamide 1 (S1) is associated with the apical and oral ganglia and, especially in the asteroids, the dorsal ciliary band. This distribution is similar to that of serotonin, with evidence for both distinct sets of peptidergic and serotonergic neurons in all species together with coexpression in some cells of Dendraster, as indicated by double labelling. These early results suggest a possible function for $\mathrm{S} 1$ in feeding and motility as well as a sensory role in association with serotonin. An interesting finding is the expression of an S1-like molecule in association with cells comprising the larval vestibule, the structure from which all adult tissues develop.

\section{Regulation of gene expression in the sea urchin embryo}

\section{J. A. Coffman and E. H. Davidson \\ Division of Biology, California Institute of Technology, Pasadena, CA 91125, USA}

In the undisturbed sea urchin embryo, cleavage of the blastomeres is invariant and gives rise to five polyclonal territories that are each defined by the larval structures to which they give rise and by unique programmes of gene expression. These territories are the aboral ectoderm, the oral ectoderm, the vegetal plate, the skeletogenic mesenchyme, and the small micromeres. Structural gene markers for four of these territories (all except the small micromeres, which participate in later development) have been cloned and characterized, and the regulatory domains of several of these genes have been mapped to the level of specific protein-binding sites. The best characterized territory-specific gene is CyIIIa, which encodes a cytoskeletal actin expressed exclusively in the aboral ectoderm. Transcription of this gene is regulated by between 8 and 12 different DNAbinding proteins that interact with approximately 20 different sites in the $2.3 \mathrm{~kb}$ regulatory domain of this gene. We have developed an automated method for purifying multiple DNAbinding proteins from nuclear extract, using specific oligonucleotide binding sites linked to a tandem series of affinity columns; with this method most of the CyIIIa transcription factors have been isolated in sufficient quantity for amino acid sequence analysis. Amino acid sequence obtained from several of the affinity purified CyIIIa transcription factors has been used to make nucleic acid probes for cloning the corresponding cDNAs. These cDNAs, and the recombinant proteins they encode, are being used to analyse the expression, regulation, and function of the regulatory proteins. We have shown previously, through chromatographic enrichment and twodimensional electrophoretic mapping, that the complexity of nuclear proteins that are candidates for being transcriptional regulators is of the order of 100 . In addition, a recent study indicates that most if not all of the CyIIIa transcription factors are present in the unfertilized egg cytoplasm, suggesting that localized activation of a subset of maternal factors is responsible for initial specification of the aboral ectoderm territory. These studies indicate that it is now possible, with the approach and techniques discussed here, to clone and characterize all the transcriptional regulatory molecules involved in early cell fate specification in the sea urchin embryo. 


\title{
The molecular control of spatial patterning in amphioxus
}

\author{
P.W.H. Holland*, N.A. Williams*, J. Garcia-Fernandez* and L.Z. Holland ${ }^{\dagger}$ \\ ${ }^{*}$ Department of Zoology, University of Oxford, Oxford, OX1 3PS \\ ${ }^{+}$Scripps Institution of Oceanography, San Diego, CA 92093, USA
}

Many genes involved in controlling spatial patterning during insect development are members of evolutionarily conserved multigene families (for example homeobox, zinc finger, Pax and Wnt genes) with very wide phylogenetic distributions. Gene disruption experiments, plus description of expression patterns, indicate that mammalian homeobox genes of the Hox class, like their insect counterparts, control region-specific cell fate during embryogenesis. To investigate whether homologous genes play similar roles during the embryonic development of protochordates, we have used PCR and library screening to search for homeobox and Wnt genes in the genome of an amphioxus, Branchiostoma floridae. Importantly, comparison of homologous patterning genes in amphioxus and vertebrates may also give insight into the genetic changes that accompanied vertebrate evolution, and into the relationship between the amphioxus and vertebrate body plans. Our PCR analyses reveal that amphioxus has Hox genes of several different subclasses, other homeobox genes of more divergent classes, and homologues of specific vertebrate Wnt genes. In situ hybridization using probes from an amphioxus $H o x$ gene of the $p b$ subclass reveals a patch of expressing cells in the most posterior mesoderm, and a more extensive region-specific domain of expression in the neural tube of amphioxus neurulae, later embryos and larvae. The patterns found are consistent with this gene being a regulator of spatial patterning during amphioxus neural tube development, but not mesoderm development. There is a defined anterior limit to expression, as with homologous genes in other taxa; comparing this position between species gives insight into homology between the amphioxus and vertebrate body plans.

\section{Development and regeneration of the nervous system in the ascidian Ciona intestinalis}

T. Bollner, P.W. Beesley and M.C. Thorndyke

Department of Biology and Department of Biochemistry, Royal Holloway, University of London, Egham, Surrey, TW20 OBX

In the context of development, regeneration is the restorative process by which tissue lost through trauma is replaced. This is achieved through re-deployment of developmental processes such as proliferation, migration and differentiation. In spite of superficial similarities these are not equivalent processes since the cells which give rise to the regenerate exist in an environment different from that of the developing system. Ciona intestinalis and other ascidians have previously been used extensively in studies of development during embryogenesis. In contrast, rather less is known about post-metamorphic development, and in particular the relationship between this process and that of regeneration. In this study of $C$. intestinalis we compare regeneration of the neural complex following total ablation with normal development of the nervous system. Developing and regenerating cells were identified by immunocytochemistry, using antisera raised against some neuropeptides and to the classical neurotransmitter GABA. Monoclonal antibodies to $C$. intestinalis neural tissue components were produced and used as markers for non-transmitter molecules. Mitotic activity was investigated through incorporation of 5bromodeoxyuridine (BrdU) revealed by a specific antibody.

During embryogenesis the larval central nervous system (CNS) is formed through neurulation and part of the neural tube comprises the primordium to the post-metamorphic (adult) CNS. During metamorphosis proliferation in this tissue gives rise to both the neural (brain) and 
glandular parts of the adult CNS. Shortly after metamorphosis substance P-, cholecystokininand GABA-like immunoreactive cells were present in the brain. The sequence in which these molecules first appear was recapitulated during regeneration. Staining patterns observed with the monoclonal antibodies indicate that regeneration follows the same pattern as normal development. It thus seems that although the micro-environment of the regenerating cells is probably different from that in normal development, the end product of both processes are much the same. The origin of the cells comprising the regenerated brain could not be fully ascertained. The BrdU experiments suggested that at least some of the new cells come from the dorsal strand, which is of neural plate origin and therefore shares the same precursors as the original cells. However, not all types of regenerating brain cells were labelled with BrdU. A possible explanation for this is that pluripotent non-neuronal cells, born prior to the ablation, transdifferentiate to neuronal cells. It is not known at present if this phenomenon is unique to regeneration nor if such a mechanism is also involved in normal brain growth.

\section{Single cell analysis of muscle cell differentiation in early Xenopus embryos}

\section{Kazuto Kato and J. B. Gurdon \\ Wellcome/CRC Institute, University of Cambridge, Tennis Court Road, Cambridge, CB2 1QR}

We have used a single-cell transplantation technique to study the importance of cell-cell interactions for muscle cell differentiation in Xenopus laevis embryos. Muscle precursor cells from early, mid- and late gastrula stages of Xenopus embryos were isolated and transplanted singly into the ventral region of late gastrula hosts. Single cells from late gastrulae differentiated into muscle when surrounded by non-muscle cells. Similar cells from early or mid-gastrulae did not, unless they were transplanted as a group of adjacent cells taken from the same region of an embryo. These results show that single embryonic cells in a tissue can complete their differentiation without interacting with their normal neighbours and that, in the case of Xenopus muscle precursor cells, they acquire this capacity at the late gastrula stage. Our results also suggest that, in addition to mesoderm induction, further cell interactions during gastrulation are important for Xenopus muscle cell differentiation. Attempts to analyse further the nature of these interactions are also discussed.

\section{Specification of cell lines in the embryo of Platynereis (Annelida: Polychaeta)}

\section{A. Dorresteijn \\ Zoologisches Institut der Johannes-Gutenberg-Universität, Saarstraße 21, W-6500 Mainz, Germany}

The unequal, spiral cleavage pattern of the polychaete, Platynereis dumerilii, creates blastomeres of different sizes. Yolk and yolk-free cytoplasm segregate and become rearranged in a very constant manner within the blastomeres prior to each cleavage. Due to the asymmetrical position of the spindle, distribution of cytoplasmic components is disproportional to the volume of the daughter blastomeres. The yolk-free cytoplasm is predominantly shunted into the blastomeres $2 \mathrm{~d}$ and $4 \mathrm{~d}$, which mark the dorsal mid-line and proliferate bilaterally symmetrically. Experimentally induced equal distribution of the yolk-free cytoplasm at first or second cleavage results in a duplication of the $2 \mathrm{~d}$ and $4 \mathrm{~d}$ cells. Such embryos develop into young worms having trunks with two dorsal and two ventral sides. This shows that cytoplasmic distribution influences the fate of cells as well as the dorsoventral pattern in the trunk.

Differentiation marker molecules specific to larval gland cells and neural cell lines of young worms allow us to trace the progenitor cells of these cell lines in early cleavage stages, and to analyse such developmental steps which imprint 'fate' in these progenitor cells. For this purpose, 
embryos were cleavage-arrested at successive cleavage stages in $0.2 \mu \mathrm{g}$ cytochalasin B per ml seawater over the same period of time needed by controls to start expression of the differentiation markers. Then, the cleavage-arrested, aged embryos underwent the same test. An antigen specific to five larval gland cells in the ventral head region of the trochophore is expressed in every cleavage-arrested stage and can even be detected in the uncleaved, aged zygote. The competence to form this antigen gradually becomes restricted to the $1 \mathrm{a}$ and $1 \mathrm{~b}$ cell lines in older stages. This shows that the essential conditions for the expression of this antigen are present in the zygote and become restricted by cleavages. The competence to form neural differentiation markers, however, is not accomplished in any of the blastomeres of cleavage stages arrested before $5.5 \mathrm{~h}$ of development. Embryos cleavage-arrested at stages older than $7 \mathrm{~h}$ express neural differentiation markers in part of the progeny of the $2 \mathrm{~d}$ blastomere $\left(2 \mathrm{~d}^{2}, 2 \mathrm{~d}^{12}, 2 \mathrm{~d}^{1121}\right.$ and $\left.2 \mathrm{~d}^{1122}\right)$. Thus the fate of the neural progenitor cells seems to depend upon the conditions which are firstly achieved after an appropriate cleavage history.

\title{
Responses of cutaneous chloride cells in the larvae of the turbot (Scophthalmus maximus) to changes in salinity and temperature during incubation
}

\author{
P. Tytler and J. Ireland \\ Department of Biological Sciences, University of Stirling, Stirling, FK9 4LA
}

Increases in temperature and salinity have been shown to challenge the osmoregulatory system of marine fish larvae by increasing diffusional permeability and the osmotic gradient. To maintain water balance, larvae adjust drinking rates and water absorption efficiency, processes which exacerbate salt loading. As yet little is known about the salt excretion processes in fish larvae. Cutaneous mitochondria-rich cells, which have a similar ultrastructure to adult branchial chloride cells, are thought to be the sites of ion excretion. In this study the effects of changing temperature and salinity during incubation of early stage larvae of turbot on various features of these putative chloride cells were investigated. In particular, changes in mitochondrial protein were estimated by measuring, in vivo, the intensity of fluorescence in cells of larvae exposed to DASMEI by confocal microscopy and computer-assisted image analysis. Both environmental variables have highly significant effects on chloride cell fluorescence, with highest levels measured at high temperature and salinity. Electron microscopy has shown concurrent changes in the size of mitochondria and the elaboration of cristae.

The application of confocal microscopy of various aspects of the ontogeny of osmoregulation in transparent larvae, including gut and pronephric function, are discussed.

\section{Temperature and muscle development in the embryos and larvae of Atlantic herring (Clupea harengus: Teleostei)}

\author{
I.A. Johnston and Z. Horne
}

Gatty Marine Laboratory, School of Biological and Medical Sciences, University of St Andrews, St Andrews, Fife, KY16 8LB

Muscle gene expression and differentiation are being investigated in the embryos and larvae of Atlantic herring (Clupea harengus L.). Eggs from spring spawning stocks have been reared at temperatures ranging from 5 to $15^{\circ} \mathrm{C}$. Larvae hatched after $8 \mathrm{~d}$ at $15^{\circ} \mathrm{C}$, and $26 \mathrm{~d}$ at $5^{\circ} \mathrm{C}$. The differentiation of the axial muscles was studied in relation to the development of other organs and tissues using light and electron microscopy. Evidence was obtained that temperature influenced the relative timing of myogenesis and the commitment of myoblasts to differentiation. Myoblasts fused to form myotubes and immature muscle fibres after $12-16 \mathrm{~d}$ at $5^{\circ} \mathrm{C}, 7-10 \mathrm{~d}$ at $8^{\circ} \mathrm{C}$ 
and $3-5 \mathrm{~d}$ at $12^{\circ} \mathrm{C}$. Following this initial period of myogenesis the number of myotomal muscle fibres remained constant until 1-2 weeks after hatching, such that muscle growth in the late embryo and yolk-sac larvae was entirely due to fibre hypertrophy. The number of presumptive myosatellite cells per $\mathrm{mm}^{2}$ muscle fibre cross-sectional area was more than twice as high in larvae reared at $8^{\circ} \mathrm{C}$ as at either $5^{\circ} \mathrm{C}$ or $12^{\circ} \mathrm{C}$. At hatching the myotomes contained a superficial monolayer of small diameter fibres surrounding an inner mass of 200-300 larger-diameter muscle fibres. The number of muscle fibres/myotomes tended to be higher with increasing rearing temperature, although the results were somewhat variable between spawning groups. Temperature was also found to influence the spatial organization and volume densities of muscle fibre organelles. Distinct isoforms of a number of the myofibrillar proteins have been identified in embryonic, larval, juvenile and adult stages. The larval muscle fibre types are gradually replaced with adult fast and slow muscles, concomitant with the development of functional gills and other changes in body morphology. Monoclonal antibodies have been raised against herring myofibrillar proteins. Metamorphosis is associated with a thickening of the layer of superficial muscle fibres and a switch in the expression of myosin light chains from larval to adult types. Future studies will investigate the influence of temperature on the relative timing of myofibrillar protein gene expression. Our results highlight the importance of temperature in directing the outcome of early development and in determining muscle phenotype, with corresponding implications for the physiological performance and survival of larval stages.

\section{Ecology and biology of Acetabularia}

\section{S. Bonotto \\ Department of Animal Biology, University of Turin, Italy}

Acetabularia (Dasycladaceae, Chlorophyta) is a giant unicellular marine alga presenting a peculiar morphological differentiation, comprising the development of a branched rhizoid at its basal end, where the nucleus is located, and the formation of several whorls and then a reproductive cap at the apex of the stalk.

In nature Acetabularia fixes to solid substrata and also adheres to artificial materials. The 22 species of Acetabularia at present recorded show optimal growth in shallow waters of tropical to temperate areas, with a clear seasonality and a manifest zonation. Acetabularia may behave as a perennial plant, the very basal part of the cell surviving in the rhizoid and regenerating a new thallus when the environmental conditions become favourable.

Although unicellular Acetabularia has complex reproductive mechanisms: (a) fusion of haploid gametes with zygote formation (the most frequent); (b) zoospore formation; (c) cyst germination; (d) germination of basal cytoplasm; (e) gamete parthenogenesis; ( $f$ ) fusion of haploid secondary nuclei. Most recent work on the biology of Acetabularia was done to understand the cytological and biochemical bases of polarity and of cellular differentiation. According to current ideas, morphological development in Acetabularia is controlled by the nucleus by means of 'morphogenic substances' assimilable to long-lived molecules of mRNA. In addition, experiments with anucleate cells, which maintain the capability of forming whorls and one (or more) cap, suggested that a differential and sequential activation of nuclear genes can be excluded. Consequently, cellular differentiation in Acetabularia would be regulated at the translational level, possibly by a spatial and temporal selection of stored mRNAs. 


\title{
The effect of temperature and food quantity on the development of Tisbe battagliai (Copepoda: Harpacticoida)
}

T.D. Williams* and M.B. Jones ${ }^{\dagger}$

${ }^{*} I C I$ Group Environmental Laboratory, Brixham, Devon, TQ5 8BA. ${ }^{+}$University of Plymouth, Drake

Circus, Plymouth, Devon, PL4 8AA

Development of nauplius to adult and the duration of the life stages of the marine harpacticoid, Tisbe battagliai, were investigated in relation to temperature and food supply.

Tisbe battagliai was raised in the laboratory from birth to adult on six different concentrations of the algae Isochrysis galbana ( 0 to $\left.3,250 \mu \mathrm{g} \mathrm{C}^{-1}\right)$ at three different temperatures $\left(15,20\right.$ and $\left.25^{\circ} \mathrm{C}\right)$. Development rates were influenced by both temperature and food concentration, rates decreasing with increasing temperature and quantity of food available. At low food concentrations, naupliar survival decreased and stage duration increased at levels dependent upon the test temperature. The importance of these environmental factors for influencing the development of copepod populations in the natural environment is discussed.

\section{POSTERS}

Plasmalemma redox chain transduces polarization of the Fucus zygote by blue light

\author{
F. Berger, A. R. Taylor and C. Brownlee \\ Marine Biological Association, The Laboratory, Citadel Hill, Plymouth, PL1 2PB and ENS Lyon, 46 \\ Allée d'Italie, 69364 Lyon Cédex 07, France
}

During the photosensitive period, a few hours after fertilization, the zygote of the marine brown alga Fucus polarises according to the prevailing light direction. The first cell division then determines a rhizoid and a thallus cell. The light path inside the zygote observed with a red laser beam indicates that germination of the rhizoid takes place on the side away from the light source which also receives the highest light intensity. Blue light is responsible for polarization. In other models, plasmalemma redox chains are involved in transduction of blue light signals. Ferricyanide (FeCY), as an electron acceptor, affects electron transport in redox chains. FeCY (1-50 mM) inhibits Fucus zygote photopolarization. Inhibition is dose-dependent. Higher concentrations $(>50 \mathrm{mM}$ ) inhibit growth only if $\mathrm{FeCY}$ is applied for periods longer than $1 \mathrm{~h}$ during the photosensitive period and in the light. Inhibition of growth was not observed in the dark. We propose that this inhibition originates from depletion of reduced equivalent in the cytoplasm. FeCY proves to be a 'physiological' inhibitor of the polarization of the Fucus zygotes probably by acting on a plasmalemma redox chain transducing the blue light signal.

\section{In the ontogenesis of Mytilus the first nervous rudiments appear in a posterior position}

\author{
M. Raineri \\ Institute of Comparative Anatomy, 16132 Genoa, Italy
}

The cerebral ganglia which develop in connection with the sense organs of the apical plate are considered the first nervous rudiments in the trochophore larva in general. In Mytilus edulis, however, neurohumors and enzymes related to their metabolism are first localized in a few cells at the posterior surface of the early trochophore. The present study investigates neurogenesis in M. galloprovincialis by a histochemical localization of acetylcholinesterase (AChE) and 
pseudocholinesterase (BuChE). The enzyme activities are first detected in two bilaterally symmetrical cells at the posterior surface of the early gastrula. Under each of them during gastrulation another histochemically stained cell develops strongly AChE-active cilia which elongate at the lateral plasma membranes of the superficial cell. In the trochophore superficial and inner cells increase in number, the former stain intensely for $\mathrm{BuChE}$, the latter for $\mathrm{AChE}$ and grow axon-like processes. These cell clusters are covered by a BuChE-active jelly-like coat and look-alike secretory-sensory ciliary organs. AChE is also associated with the basal plasma membranes of the trochoblasts and, in the late trochophore, with cells localized at the base of the apical tuft and anteriorly in two bilaterally symmetrical clusters. A row of AChE-active cells runs along each side under the developing velum to connect anterior and posterior cell clusters which differentiate into nervous ganglia in the veliger.

\title{
Tyrosine phosphorylation during meiosis reinitiation in clam oocytes
}

\author{
H. Abdelmajid, P. Rivailler, P. Guerrier and S. Krantic
}

UMR 49, Ecole Normale Supérieure de Lyon, 46 Allée d'Italie, 69364 Lyon Cédex 07, France

The neurohormone serotonin (5-hydroxytryptamine, 5-HT) triggers meiosis reinitiation in oocytes of the pelecypod molluscs, Spisula solidissima and Ruditapes philipinarum. Using antiphosphotyrosine antibodies, we found that $5-\mathrm{HT}$ promoted tyrosine dephosphorylation of $\mathrm{p} 34^{\text {cdc2 }}$ within $15 \mathrm{~min}$ in the oocytes of Spisula which undergo full maturation, but not in those of Ruditapes which arrest in metaphase 1 and maintain a high level of histone H1-kinase activity. However, in both species 5-HT triggers a transient tyrosine phosphorylation of a $42 \mathrm{kD}$ anti-MAP positive protein, which begins within $5 \mathrm{~min}$ of 5 -HT addition and stops $10 \mathrm{~min}$ later.

Absence of external calcium blocks meiosis reinitiation. Under these conditions there is neither dephosphorylation of $\mathrm{p} 34^{\mathrm{cdc} 2}$, nor phosphorylation of the $\mathrm{p} 42 \mathrm{MAP}$ protein, while two other proteins (72 and $123 \mathrm{kD}$ ) become phophorylated on tyrosine.

The different behaviour of Spisula and Ruditapes oocytes suggests that MPF may occur even in the absence of tyrosine dephosphorylation of $\mathrm{p} 34^{\mathrm{cdc} 2}$ and that this dephosphorylation may be required for achievement of the metaphase-anaphase transition.

\section{Release from the prophase block of Patella vulgata oocytes: roles of pH and PKC}

\section{Gobet, F. Berger, P. Guerrier and S. Krantic}

UMR 49, Ecole Normale Supérieure de Lyon, 46 Allée d'Italie, 69364 Lyon Cédex 07, France

In the gonad, oocytes of the mollusc prosobranch Patella vulgata are arrested in prophase of the first meiotic division. Meiosis reinitiation can be induced by a cytoplasmic alkalinization provided by weak bases like ammonia and amiloride or after injection of alkaline buffers. The alkalinization produced by ammonia leads to activation of MPF and increase of H1 kinase activity.

The phorbol ester PMA, which is known to activate PKC, can also trigger $\mathrm{H} 1$ kinase activation and release from the prophase block. The specificity of this action was shown using an inactive analogue of PMA (PMA-met) and a novel potent and selective inhibitor of PKC (GF 109203X). PMA is inactive in the absence of sodium in the external medium or when the intracellular $\mathrm{pH}$ is decreased by a weak acid like sodium acetate. Finally, PMA seems to induce a very small change in intracellular $\mathrm{pH}$ as measured with BCECF. On the other hand, GF109203X can inhibit the response of prophase-arrested oocytes to ammonia and amiloride. Our results suggest that both cytoplasmic alkalinization and PKC activation are required for the induction of meiosis resumption in Patella oocytes. 


\title{
Cell cycle dynamics of cdc2-like proteins in a dinoflagellate
}

\author{
J. Wong
}

Marine Biological Association, The Laboratory, Citadel Hill, Plymouth, PL1 2PB

Dinoflagellates have many cellular characteristics distinct form the rest of the eukaryotes. The majority of the species are characterized by the absence of histones and nucleosomes, the presence of a permanent nuclear envelope, permanently condensed chromosomes and an extracellular spindle. An improved method is reported here which can produce a population of Crypthecodinium cohnii at a higher degree of synchrony $(\sim 80 \%)$ than previously reported. Flow cytometry was also used for the first time to analyse the cell cycle progression of this population. The two-cell cyst sub-population required $10 \mathrm{~h}$ at $28^{\circ} \mathrm{C}$ to complete the first cell cycle on release from cysts. Using an antibody against the PSTAIRE peptide, I have also identified at least three PSTAIRE-containing polypeptides in a synchronous population of $\mathrm{C}$. cohnii, two of which are apparently cell-cycle-stage specific. In addition, none was found to be $34 \mathrm{kD}$ in size, unlike the control (baculovirus-expressed human p34). Current experiments are trying to establish the function of these polypeptides by $\mathrm{p}^{1} 3^{\text {suc1 }}$ bead precipitation and histone kinase assay (in a histone-less organism). 